\title{
Development of Segment Specificity in Identified Lineages of the Grasshopper CNS
}

\author{
Karen J. Thompson ${ }^{\mathrm{a}}$ and Melody V. S. Siegler \\ Department of Biology, Emory University, Atlanta, Georgia 30322
}

The purpose of this study was to determine the factors underlying differences in population size and composition between segmentally homologous neuronal lineages. The segmental median neuroblasts (MNBs) of grasshoppers are identified stem cells that each produce a midline group of neurons. We traced the embryonic development of the group in two disparate segments, counting MNB progeny and profiles of dying cells in fixed and stained preparations of staged embryos. In the metathoracic segment (T3), about 95 MNB progeny survive embryonic development, whereas in the next posterior segment, the first abdominal (A1), only about 60 survive. In T3, the MNB arises at $29 \%$ of embryogenesis and dies at $78 \%$, whereas in A1 the MNB arises at $30 \%$ and dies at $73 \%$. In T3, the number of MNB progeny initially increases at a steady rate, 10 cells being added per $5 \%$ of embryogenesis. Between $70 \%$ and $78 \%$ growth tapers off; although the T3 MNB continues to divide, cells die at the same time, specifically removing last-born progeny. By contrast, in A1 the MNB progeny increase in two phases, one from $30 \%$ to $45 \%$ and the other from $60 \%$ to $73 \%$, again at the rate of 10 cells per $5 \%$. Between the two phases, the number of A1 progeny is stable. The A1 MNB continues to divide, but cells die at the same time, specifically removing earlier-born progeny. The episodes of cell death in A1 and T3 coincide with embryonic molts, and thus may be hormonally triggered. Cell death is greater in A1 than T3, accounting for most of the difference in population size. The difference in MNB longevity makes a lesser contribution. The present data, together with corollary anatomical data (Thompson and Siegler, 1991), support the hypothesis that progeny fated to become certain neuronal types are selectively removed from the two MNB lineages: intersegmental interneurons from T3 and efferent neurons and local interneurons from $A 1$.

[Key words: cell death, embryonic development, grasshopper, insect, neuroblast, segmental homology]

Studies addressing the issue of diversity in neuronal lineages have confirmed that numerous cell types can be produced by a single stem cell in lineages that may be variable or deterministic,

\footnotetext{
Received June 29, 1992; revised Jan. 29, 1993; accepted Feb. 1, 1993.

We thank Drs. Jeffrey P. Conn, Hilary Ellis, and Barry Yedvobnick for their helpful comments on the manuscript. This research was supported by grants from NIH, the Whitchall Foundation, and NSF to M.V.S.S.

a Correspondence should be addressed to Karen J. Thompson, at her present address: Department of Biology, Agnes Scott College, 141 East College Avenue, Decatur, GA 30030.
}

Copyright (C) 1993 Society for Neuroscience $0270-6474 / 93 / 133309-10 \$ 05.00 / 0$ and that intrinsic and extrinsic mechanisms may play roles in either case (see McConnell, 1991, for review). Much still remains to be learned about how developmental processes control neuronal number and the acquisition of neuronal phenotype.

In the present study we have taken advantage of attributes of the grasshopper to examine the production of specific lineages by identified neuroblasts. The grasshopper is one of very few animals with both identifiable neural stem cells and identifiable adult neurons. Individual neuroblasts and neurons have the same location and propertics from cmbryo to embryo or adult to adult. Neuroblasts and their progeny are large and accessible for study with cellular precision. Previous work has established the composition of the neuroblast arrays in embryos and the pattern of divisions that they undergo (Wheeler, 1893; Bate, 1976; Bate and Grunewald, 1981; Doe and Goodman, 1985). In addition, a tradition of intracellular neuroethological investigation has resulted in extensive documentation of the properties of numerous identified adult neurons. Thus, neurodevelopmental studies in grasshoppers can directly examine the production of known neurons by identified stem cells.

Insects that undergo a complete metamorphosis, such as flies and moths (e.g., Drosophila and Manduca), show substantial postembryonic neurogenesis associated with the remodeling of the animal from larva to pupa to adult. This contrasts with the grasshopper and other hemimetabolous insects, which do not undergo complete metamorphosis. In grasshoppers the cells of the ventral nerve cord are generated embryonically, such that upon hatching the neuroblasts have all died, the nervous system is postmitotic, and there is no evidence that more neurons are born (Bate, 1976; Bate and Grunewald, 1981; Shepherd and Bate, 1990).

In grasshopper embryos, neuroblasts undergo repeated asymmetrical divisions to produce from a few to more than 100 neural progeny (Bate, 1976). A neuroblast divides to produce a neuroblast and a smaller ganglion mother cell. The ganglion mother cell undergoes a terminal symmetrical division to produce two ganglion cells, which differentiate to become neurons. The segmental ganglia of the ventral nerve cord are thus composed of groups or families of lineally related neurons, each resulting from the divisions of a single neuroblast.

The median neuroblast (MNB) is identifiable by its size, shape, and position in each segment of grasshopper embryos. The progeny of the MNB remain closely clustered around it during development (Goodman and Spitzer, 1979; Goodman and Bate, 1981). The clustering of these midline progeny persists after the death of the neuroblast and throughout the life of the insect. In addition, the midline neurons are physically separate from the other groups of developing neurons during embryogenesis and 
from other neurons in the adult CNS. Thus, the MNB and its offspring are readily located with no need for a cell-specific marker.

In adult grasshoppers these midline neurons have been well studied and are known as the dorsal unpaired median, or DUM, neurons. Many of the more than 90 neurons in the metathoracic segment (T3) have been identified with respect to morphology, physiology, role in behavior, and neurotransmitter type (Evans and O'Shea, 1978; Hoyle, 1978; Watson, 1984; Siegler et al., 1991; Thompson and Siegler, 1991; Stevenson et al., 1992). Their properties separate them into one of three classes of neuron: efferent, local, or intersegmental. Evidence to date suggests that the lineage of the MNB in T3 is determinate, but the question of the relative contributions of intrinsic or extrinsic mechanisms in formation of the lineage is not settled (Goodman and Bate, 1981; Taghert and Goodman, 1984; Doe et al., 1985; Thompson and Siegler, 1991).

The grasshopper embryo has segmentally reiterated arrays of neuroblasts in a pattern that is essentially the same in every thoracic or abdominal segment (Bate, 1976): 30 bilateral pairs, in seven rows of two to six neuroblasts, and the single posterior MNB studied here. This segmental uniformity is in marked contrast to the regional specializations of segments in the adult. For example, the segmental ganglion in the thorax that controls the jumping legs and hindwings (metathoracic, T3) has approximately 2000 nerve cells, while small abdominal ganglia in segments that do not bear legs or wings have only about 400 neurons.

Of relevance for the present study is the observation that the composition of the lineages produced by the MNBs varies among segments. This variation allows us to ask how homologous MNBs can produce different lineages. Here we show that temporally and segmentally distinct episodes of cell death appear to be the major mechanisms accounting for differences in the number and complement of surviving progeny of serially homologous neuroblasts. Furthermore, it appears that the timing of cell death is correlated with two of the four embryonic molts.

\section{Methods}

Staging of embryos. The embryonic development of Schistocerca americana, the grasshoppers used in our studies, takes $20 \mathrm{~d}$ at $33^{\circ} \mathrm{C}$. Embryos were staged according to percentage of embryogenesis (Bentley et al., 1979). The time at which eggs are laid is $0 \%$ (eggs are fertilized as they pass down the oviduct), and $100 \%$ is the time of hatching. Therefore, a $50 \%$ embryo is halfway through embryonic time. Embryos were either assigned to one of the $5 \%$ incremental stages of Bentley et al. (1979) or, if clearly between stages, assigned to the midpoint between them. For the quantitative analyses in this study, embryos were grouped together in $5 \%$ stages. Our estimated error in staging is $\pm 1 \%$.

Toluidine blue staining. Neuroblasts and neuronal cell bodies were stained with toluidine blue dye (Altman and Bell, 1973). Different times and protocols were found to be appropriate for embryos and adults. Briefly, embryos of $25-65 \%$ were dissected to expose the dorsal surface of the developing nerve cord in saline containing (in mM) $150 \mathrm{NaCl}, 3$ $\mathrm{KCl}, 2 \mathrm{CaCl}_{2}, 1 \mathrm{MgSO}_{4}$, and 5 TES ( $N$-tris[hydroxymethy]-methyl-2aminoethane-sulfonic acid), and adjusted to $\mathrm{pH}$ 7.2. They were stained in situ with toluidine blue dye for $15 \mathrm{~min}$ at room temperature, and then destained with Bodian's II fixative for 1-5 min, dehydrated with 2 min steps in $90 \%, 95 \%$, and twice in $100 \%$ ethanol, cleared in methyl salicylate, and mounted in Canada balsam. The treatment of embryos older than $65 \%$ involved dissecting embryos as above except sometimes the developing nerve cords were removed from the embryos before staining 20-30 min at room temperature, and then dehydrating, clearing, and mounting as above. For adults the ganglia were removed from the animals and stained for 30-45 min at room tempcraturc, or $15 \mathrm{~min}$ at room temperature and $15 \mathrm{~min}$ at $50^{\circ} \mathrm{C}$. Preparations were destained in Bodian's II fixative for 15-30 min, dehydrated in 5-10 min steps, cleared, and mountcd as above. These procedures produced ganglia containing neurons with dark nuclei and an outer rim of dark cytoplasm. Glial and sheath nuclei were only lightly stained. The dark staining of neuronal nuclei was an advantage because it helped to reveal deep neurons in whole-mounts. Other preparations were treated with a 2 min prefix in Bodian's II:saline (1:1) for $2-5 \mathrm{~min}$ before staining. With this treatment the nuclei of the neurons were unstained, but the neuronal cytoplasm was stained. Glial and sheath cell nuclei were completely unstained. Embryonic preparations were examined in bright-field or DIC optics at 945-1500 $\times$ and adult preparations at $600 \times$. Drawings were made using a drawing tube attached to a compound microscope. Cells were counted from drawings of whole-mounts of toluidine blue-stained material. Pyknotic nuclei were counted directly from whole-mounts, at $1500 \times$ with DIC optics.

Feulgen staining for whole-mounts and sections. Embryos were separated from the yolk and gut and fixed in nonalcoholic Bouin's fluid. They were treated with $5 \mathrm{~N} \mathrm{HCl}$ for $30 \mathrm{~min}$, and stained in Schiff reagent (Sigma) for $2 \mathrm{hr}$ in the dark. They were then bleached with sulfrous acid solution $\left(0.05 \mathrm{~N} \mathrm{HCl}, 0.4 \% \mathrm{Na}_{2} \mathrm{~S}_{2} \mathrm{O}_{5}\right)$ and dehydrated through a graded series of alcohols to methyl salicylate for viewing in whole-mount or embedding in Epon.

GABA immunohistochemistry and sulfide silver staining. Whole metathoracic ganglia were stained for GABA-like immunoreactivity following the protocol of Witten and Truman (1991). The antiserum was obtained from Chemicon, Inc. Ganglia were stained for heavy metals to reveal octopamine-containing neurons, following the protocol of Siegler et al. (1991).

Epon sections. Selected embryos were taken from methyl salicylate to Epon for sectioning at a thickness of $4-8 \mu \mathrm{m}$, and stained with a mixture of methylene blue and toluidine blue. Some ganglia had been fixed in Bouin's fixative and dehydrated. Others had been stained with the Feulgen method. See Thompson and Siegler (1991) for details of sectioning.

\section{Results}

\section{Properties of the adult MNB lineage groups}

The segmented nature of the adult metathoracic ganglion is evinced by the separate clusters of DUM neurons belonging to segments T3, A1, A2, and A 3 (Fig. 1a). The MNB lineage group in $\mathrm{T} 3$ contains approximately 93 neurons $(92.5, \mathrm{SD} 3.8, N=$ 21 ), while the MNB lineage group in A1 contains approximately 60 neurons $(58.8, \mathrm{SD} 2.3, N=39$ ) (Fig. $1 b, c)$. Among the progeny of the MNB in T3 are some 20 efferent DUM neurons. In adult grasshoppers these neurons have large cell bodies (50-80 $\mu \mathrm{m}$ in diameter), a single primary neurite that bifurcates at the midline, roughly symmetrical branches, and bilateral axons that exit in the lateral nerves to supply peripheral targets (Figs. $1 b$, $2 a$ ). In scgment $\mathrm{A} 1$, there are only three efferent DUM neurons. These are similar in shape to those in T3, but are located more posteriorly, in the Al region of the metathoracic ganglionic mass (Figs. 1c, 2a).

Our recent studies have shown that in addition to the efferent DUM neurons, in T3 the MNB lineage group comprises two types of spiking interneurons, local and intersegmental (Fig. $2 b, c$; Thompson and Siegler, 1991). They have cell bodies that are much smaller than those of the efferent DUM neurons; most are $15-20 \mu \mathrm{m}$ in diameter. The interneurons have single primary neurites with bifurcations at the midline and roughly symmetrical fine branches. The local DUM interneurons have branches that curve anteriorward bilaterally in the neuropil to terminate in the primary auditory neuropil (Fig. $2 b$ ). These neurons are excited by sound. By contrast, the intersegmental DUM interneurons have thin paired axons extending at least to the mesothoracic (T2) ganglion in the antcrior connectives (Fig. 2c) and numerous fine branches in the dorsal neuropil (not shown). These neurons respond to sensory stimuli and motor activity associated with flight. The A1 lineage group also contains local and intersegmental interneurons. The local interneurons are 

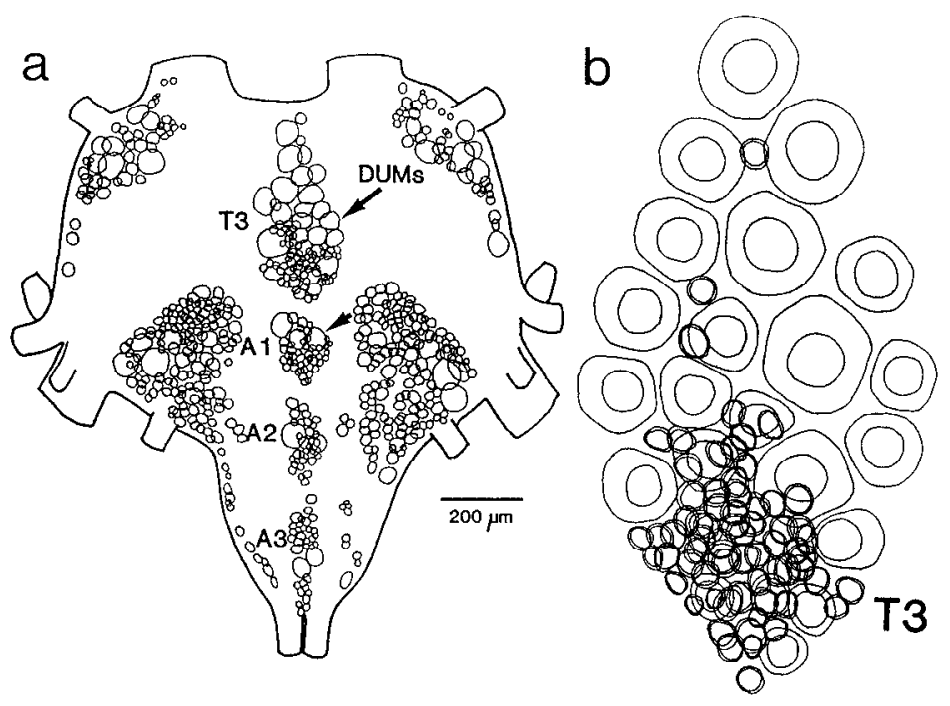

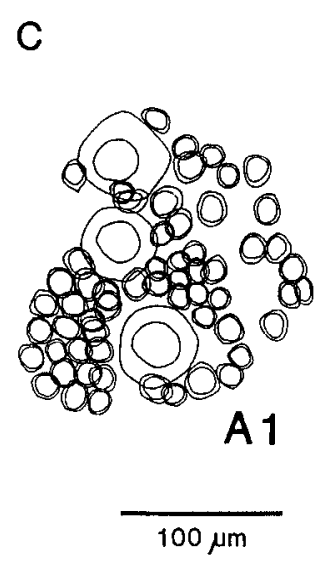

Figure 1. Neuronal cell bodies of the dorsal and dorsolateral surfaces of the adult metathoracic ganglion $(a)$, and groups of DUM neuron cell bodies in $\mathrm{T} 3(b)$ and A1 (c). The groups of DUM neurons are located in the ganglionic cortex on the dorsal midline and are separate from the cell bodies of more lateral neurons in the fused metathoracic ganglionic mass $(a)$. The groups of DUM neurons contain 20 large cell bodies and 70-80 small cell bodies in segment T3, but only 3 large cell bodies and 55-60 small cell bodies in segment A1. The illustrations are camera lucida drawings from toluidine blue-stained whole-mounts of three different adult metathoracic ganglia (nuclei were not drawn in $a$ ). similar to the auditory type of T3 (Fig. 2b). The intersegmental interneurons in $\mathrm{Al}$ are also similar to those in T3 (Fig. 2c). The neurites of the A 1 intersegmental interneurons travel along with those of T3 for some distance and they likewise send paired axons to the mesothoracic ganglion (K. J. Thompson and M. V. S. Siegler, unpublished observations).

Efferent DUM neurons in thoracic and abdominal segments have been shown to contain octopamine and to modulate tension in skeletal or visceral muscles (Evans and O'Shea, 1977, 1978; Evans and Siegler, 1982; Lange and Orchard, 1984; Morton and Evans, 1984; Malamud et al., 1988; Whim and Evans, 1988, 1989). Sulfide silver staining labels octopamine-containing neurons in insects (Siegler et al., 1991). With this technique, about 20 cell bodies are stained in $\mathrm{T} 3$ and three are stained in cach of the abdominal ncuromercs (T3 and A1 in Fig. 3c,d). The stained neurons correspond to the efferent DUM neurons with large cell bodies. The DUM interneurons do not stain for octopamine but instead show GABA-like immunoreactivity (Fig. $3 a, b$; Thompson and Siegler, 1989; Siegler et al., 1991; Stevenson et al., 1992).

\section{Embryonic production of $M N B$ progeny}

In embryos the segmental MNBs are readily identifiable in all thoracic and abdominal segments. The MNBs and their progeny are located on the midline, posteriorly in the segments; usually the MNB is found slightly posterior to the ventral ectodermal infolding at the back of its segment. For the greater part of embryogenesis, the MNBs and their progeny are also dorsally separated from the bilateral sheets of ventral neuroblasts and their groups of progeny (see Fig. 7a). The progeny of the MNBs remain tightly clustered and in close association with the neuroblast. The MNB in T3 of Schistocerca americana is first apparent just prior to $30 \%$, at a stage estimated to be $29 \%$. The MNB in A1 appears very close to $30 \%$, thus slightly later than the MNB in T3.

Once the MNBs appear, they begin proliferation. The MNB lineages are apparent as groups of cells on the posterior neuromere midline throughout embryogenesis (development of the T3 lineage is shown in Fig. 4). In the early stages of proliferation, the primary neurites of the differentiating DUM neurons extend anteriorly from the group (Fig. 4, 35\%, 40\%), but, as neurogenesis proceeds, the group expands to cover the neurites (Fig. 4 ,
$50 \%$ ). Then, as more cells are added, the lineage group assumes a rounded or triangular shape that may cover the MNB (Fig. 4, $60 \%, 70 \%$ ). The members of the lineage group are piled on top of each other until approximately $55-60 \%$, when they appear as a monolayer of tightly packed cells whose profiles fit together with little extracellular space. As cells continue to be added to the lineage a second or third partial layer sometimes is present. When the complete MNB lineage group in T3 has been produced, five or six of the cell bodies are larger than the rest, and these are usually anterior in the group (e.g., Fig. 4, 70-80\%). This contrasts to the composition of the group in adults where 20 cell bodies are clearly of larger diameter. Analysis of postembryonic development shows that selective enlargement of many efferent DUM neurons occurs in larval stages (Thompson and Siegler, unpublished observations). By approximately $78 \%$ of embryogenesis the MNB is no longer present in T3, but the MNB of A1 degenerates approximately 5\% earlier, at $73 \%$. In some preparations the dying MNB was manifest as an aggregation of small, dense droplets (see Fig. 7c).

There is minor variation among animals in the location of the MNB of T3 relative to its progeny, in the overall shape of the group of cells, and in the group's position relative to the other groups of cells in the developing ganglion at a given stage.
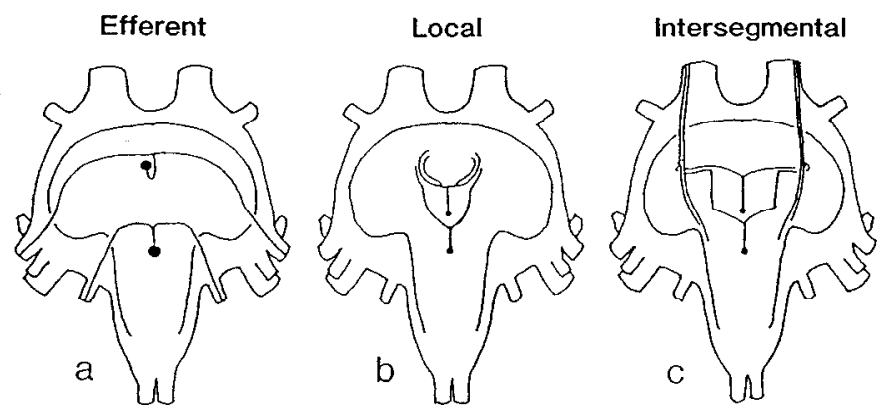

Figure 2. Neuronal cell types in the lineages of the T3 and A1 MNBs. The primary structure of each neuronal type is diagrammed within the adult metathoracic ganglion, dorsal view (anterior is up). Efferent DUM neurons with large cell bodies $(a)$, local DUM interneurons with small cell bodies $(b)$, and intersegmental DUM interneurons with small cell bodies and paired ascending axons $(c)$. T3 segmental neurons are upper, Al neurons are lower in each part. 

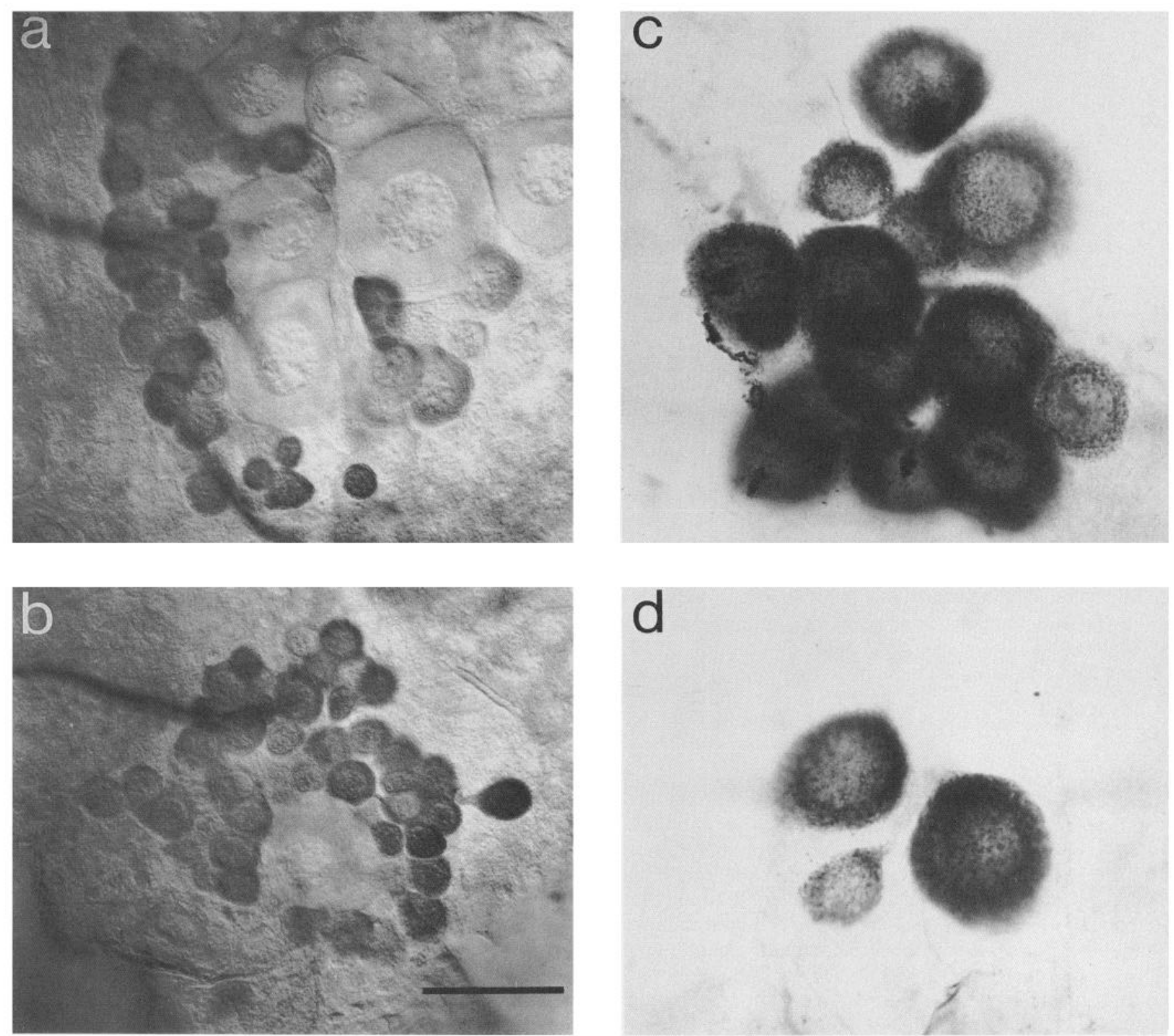

Figure 3. Differential staining of DUM interneurons and efferent DUM neurons. DUM interneurons label with antibodies to GABA in T3 (a) and in $\mathrm{Al}(b)$; efferent neurons stain positive for octopamine with sulfide silver in $\mathrm{T} 3(c)$ and $\mathrm{A} 1(\mathrm{~d})$. The interneurons have small cell bodies while the efferent neurons have large cell bodies. Unstained neurons with large cell bodies are evident in the groups photographed in $a$ and $b$. Scale bar, $50 \mu \mathrm{m}$.

However, at comparable stages of development, MNB groups always have close to the same number of cells (see below), have a posteriorly located MNB, and are separated from other groups of developing neurons in the ganglion.

Similar to the T3 group of developing neurons, the A1 group is a tight cluster of cells (examples are Figs. 8, 9). Its segmental location is between the group of MNB progeny in T3 and the group of MNB progeny in A2 (e.g., Fig. 5, 45-75\%). However, during the later stages of embryogenesis, after the MNBs have disappeared in T3 and A1, the MNB lineage groups on the dorsal surface temporarily become less spatially isolated from each other and from lateral cells (Fig. 5, 85\%, 95\%, $1^{\text {st }}$ ). By the second or third postembryonic larval stage, the separation of groups is again very clear, similar to the adult (not shown). Because of ganglionic fusion (see below), in some preparations it was difficult to ascertain the boundaries of the midline groups, es- pecially of $\mathrm{A} 1$, after $80 \%$. For many, however, despite the close apposition a clear fine boundary delineated the groups. The unstained space in toluidine blue-stained preparations is occupied by glial cytoplasm, which wraps groups of developing neural progeny during embryonic development (Doe and Goodman, 1985). A similar sheet surrounds neuronal cell body groups in adult grasshopper ganglia (Siegler and Pousman, 1990a,b; Thompson and Siegler, 1991).

During embryonic development of the metathoracic ganglion, the four component neuromeres fuse ( $\mathrm{A} 1$ to $\mathrm{T} 3 \mathrm{at}$ approximately $45-50 \%, \mathrm{~A} 2$ to $\mathrm{A} 1$ at approximately $65 \%$, and $\mathrm{A} 3$ to $\mathrm{A} 2$ at approximately $70 \%$ ), the ganglion expands in volume, and the relative position of cell body groups on the dorsal surface changes (Fig. 5). These changes are associated with addition of more neurons due to proliferation, loss of neurons due to cell death, expansion of neuropil, and enlargement of neuronal cell bodies. 


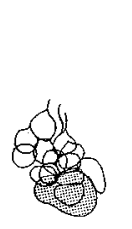

$35 \%$

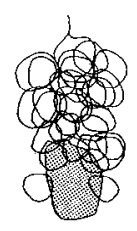

$40 \%$

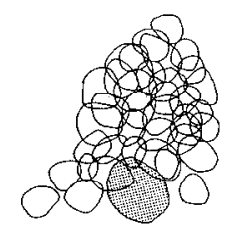

$45 \%$

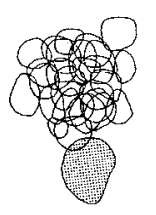

$50 \%$
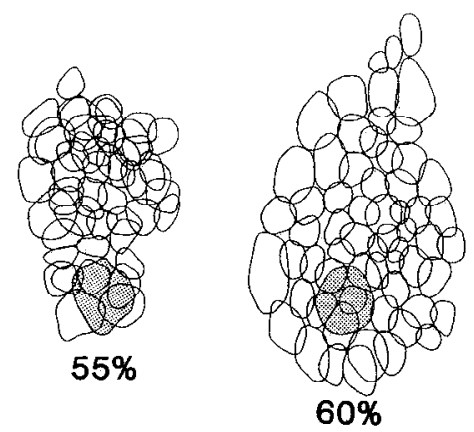

Figure 4. Embryonic proliferation of neural progeny by the MNB in the T3 segment. Camera lucida drawings show the appearance of the group at each 5\% from $35 \%$ to $80 \%$ of development in fixed preparations stained with toluidine blue. The cell bodies of neural progeny are outlined (nuclei are not indicated), and the posterior MNB is stippled. The MNB is no longer present at $80 \%$; anterior is up.

\section{Population profiles differ between $T 3$ and A1}

The production of neural progeny by the MNBs in 13 and $A 1$ was followed throughout embryogenesis, by determining the number of developing neurons associated with the MNBs in 70 T3 lineage groups and in $53 \mathrm{~A} 1$ lineage groups (Fig. 6a,b). Plots of the number of cells versus embryonic stage revealed pronounced differences in the population profiles between the T3 and A1 groups. In T3 (Fig. 6a) the number of progeny increased steadily after the appearance of the MNB. Toward the end of the period of proliferative activity, after $70 \%$, the rate of increase in the number of progeny declined slightly. The slope of a linear regression line was calculated for the time of steady increase, from $28 \%$ to $70 \%$ for $\mathrm{T} 3$. In $\mathrm{T} 3$, the number of progeny in the midline group increased at a rate of 10 cells per $5 \%$ of development during this time. The slight reduction in rate toward the end of the life of the MNB caused a deviation from linear, a phenomenon we have investigated further (see below), but for the calculation here the last $8 \%$ of development was excluded (Fig. 6a). The deviation at the end of the lineage resulted in approximatcly five fewer progeny bcing added to the T3 group than would have been predicted if the rate of increase had remained constant until the death of the MNB. After the death of the MNB at $78 \%$, the number of progeny remained stable, throughout the rest of embryonic development as well as in first through fifth stage larvae and adults.

In $\mathrm{A} 1$, the population profile did not convey a continual increase (Fig. $6 b$ ). The increase in the number of progeny in the A1 lineage group occurred in two phases, one at the beginning of MNB proliferation and the other at the end. No decline in rate occurred at the end. In the middle of the production of the A1 lineage, a plateau occurred during which time there was no increase in the size of the group. The plateau took place from $45 \%$ to $60 \%$ of development. During the plateau, the cell counts remained stable at a mean value of 29 cells. During the two phases of growth the Al midline group enlarged at a rate very similar to that of the T3 group, at 9.8 cells per $5 \%$ of embryogenesis. This value was obtained by calculating the slope of the linear regression lines for the phases from $30 \%$ to $45 \%$ and from $60 \%$ to $75 \%$. Had growth of the population in A1 instead occurred at this rate throughout, without an intcrvening plateau, some 25 more neurons would have been produced in the A1 lineage. After $73 \%$, when the MNB died in A1, the population size was stable. The size of the population in Al was determined in $80-100 \%$ embryos, larvae, and adults.

Thus, two temporally and segmentally distinct embryonic periods were identified during which the population increase in the MNB lineages deviated from linear growth. In A1, from $45 \%$ to $60 \%$ of embryogenesis, population growth ceased. In T3, from $70 \%$ to $78 \%$ of embryogenesis, population growth slowed slightly, to reach the final population size. These effects could have been due to cessation of mitotic activity by the MNB in $\mathrm{A} 1$ and slowing the rate of divisions of the MNB in T3, or to cell death accompanying ongoing divisions of the MNBs, or both.

\section{Evidence for cell death in both lineages}

Evidence that cell death is responsible for deviations from linear growth was obtained by counting pyknotic nuclei in the T3 and A1 lineage groups. These condensed nuclei stained substantially darker than any other component of the embryo, either in sections or in whole-mounts (Fig. 7a,b). The largest were $7 \mu \mathrm{m}$ in diameter and circumscribed by a distinct plasma membrane. As degeneration progressed the profiles appeared as clumps of many smaller, condensed droplets, and had indistinct membranes. Counts of pyknotic nuclei revealed pronounced differences in the time course and magnitude of cell death between the two groups. In the T3 group, scant cell death occurred between $30 \%$ and $60 \%$ of embryogenesis (Figs. $6 c$, $8 a$ ). Cell death then increased, with the maximum pyknotic nuclei evident at $70 \%$. In the Al group, little cell death occurred between $30 \%$ and $40 \%$ or after $60 \%$ of embryogenesis (Fig. $6 d$ ). Cell death 
Figure 5. Embryonic formation of the metathoracic ganglion. During embryonic development of the ganglion, the four component neuromeres fuse, the ganglion expands in volume, and the relative position of cell body groups changes (dorsal views). Black areas indicate the regions of neuronal cell bodies, and the percentage of embryogenesis or larval stage is indicated above each drawing. T3, $A 1, A 2$, and $A 3$ indicate the neuromeres; some DUM groups are indicated by arrowheads.
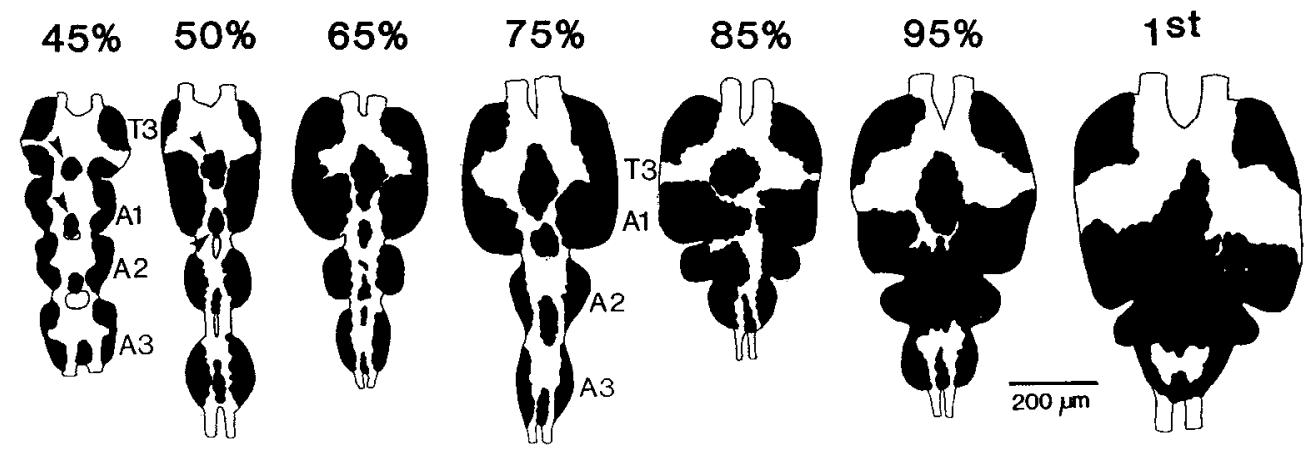

peaked between $45 \%$ and $55 \%$, with the maximum pyknotic nuclei evident at $50 \%$. The peak of cell death was substantially larger in A1 than in T3, consistent with the smaller final population size of the A1 group.

The peak of cell death in Al coincided with the plateau in population growth between $45 \%$ and $60 \%$ of embryogenesis (Fig. $6 b, d)$. At this time, the A1 group was tightly clustered. Dying cells were usually found superficially at the anterior and lateral regions of the group, but were also scattered throughout (Figs. $8 a, 9 d-f)$. The Al MNB continued to divide, apparently adding progeny at the same rate as they were dying since the population maintained roughly 29 members. The Al MNB was observed in anaphase or telophase in several preparations of $45-60 \%$ stages. By contrast, in the T3 group where MNB divisions also continued, there was scant evidence of cell death during these stages (Figs. $6 c, 8 a$ ).

The peak of cell death in T3 coincided with the slowing of population growth from $70 \%$ to $80 \%$ of embryogenesis (Fig. $6 a$, c). At this time, the T3 group was spatially extended (Fig. $8 b$ ).
The MNB and its immediate progeny in many preparations appeared plainly as an elongated strip packed among the earlierborn differentiating neurons. The pyknotic nuclei were located consistently at the distal end of this strip, away from the MNB (Figs. $7 b, 8 b, 9 a-c$ ). The cells in the strip that are closest to the MNB are the ganglion mother cells, next are the ganglion cells, and most distant are those progeny that have begun to differentiate into neurons. It thus appears that the progeny were dying near the time when, if they had survived, their cell bodies would have begun to enlarge; cell body enlargement is one indicator of neural differentiation. During this time, few or no dying cells appeared in the $\mathrm{A} 1$ group (Fig. $8 b$ ). The T3 MNB continued to divide at least until $75 \%$, as evidenced by mitotic profiles of the MNB. Degeneration of the MNB itself at $78 \%$ was associated with the appearance of a clump of several small condensed droplets of various sizes (Fig. 7c).

The relative time courses of $\mathrm{T} 3$ and $\mathrm{A} 1 \mathrm{MNB}$ activity and episodes of cell death in the $\mathrm{T} 3$ and $\mathrm{Al}$ lineage groups are summarized in Figure 10. Pyknotic profiles characteristic of cell
Figure 6. Population and cell death profiles for the lineages of the T3 and A1 MNBs during embryogenesis. In $a$ and $b$ the number of neural progeny (mean $\pm \mathrm{SD}$ ) in the lineage groups of the T3 $(a)$ and Al $(b)$ MNBs is plotted against embryonic stage. Abscissas, percentage of embryonic development; ordinates, total number of neuronal progeny. At least four but typically eight preparations were counted for each point. Regression lines for the periods of linear growth are superimposed on the data points in each graph. In $a$, for the T3 group, the line plotted is the firstorder linear regression with a slope of 10.0 cells $/ 5 \%$ of development from $28 \%$ to $70 \%$. In $b$, first-order linear regressions are plotted for the two periods of embryonic development during which the Al group enlarged, from $30 \%$ to $45 \%$ and from $60 \%$ to $73 \%$. The slope of the regression in $\mathrm{A} 1$ is 9.8 cells $/ 5 \%$. Between those two periods the population plateaued. In $c$ and $d$ the number of pyknotic nuclei (mean $\pm \mathrm{SD} ; N=4$ embryos at each stage) in the lineage groups of the T3 $(c)$ and Al $(d)$ MNBs is plotted against embryonic stage (dashed lines). Abscissas, percentage of embryonic development; ordinates, number of pyknotic nuclei.
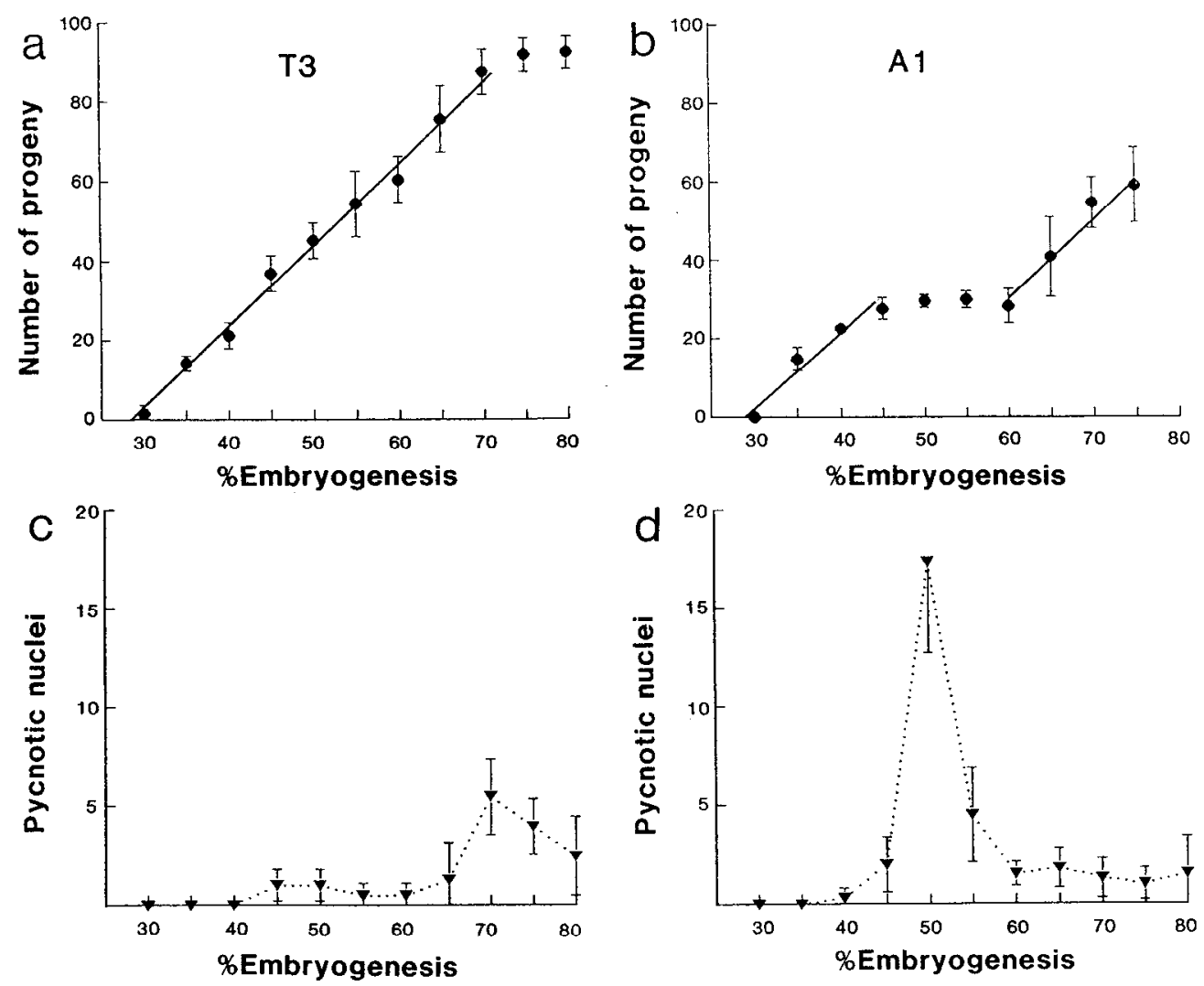

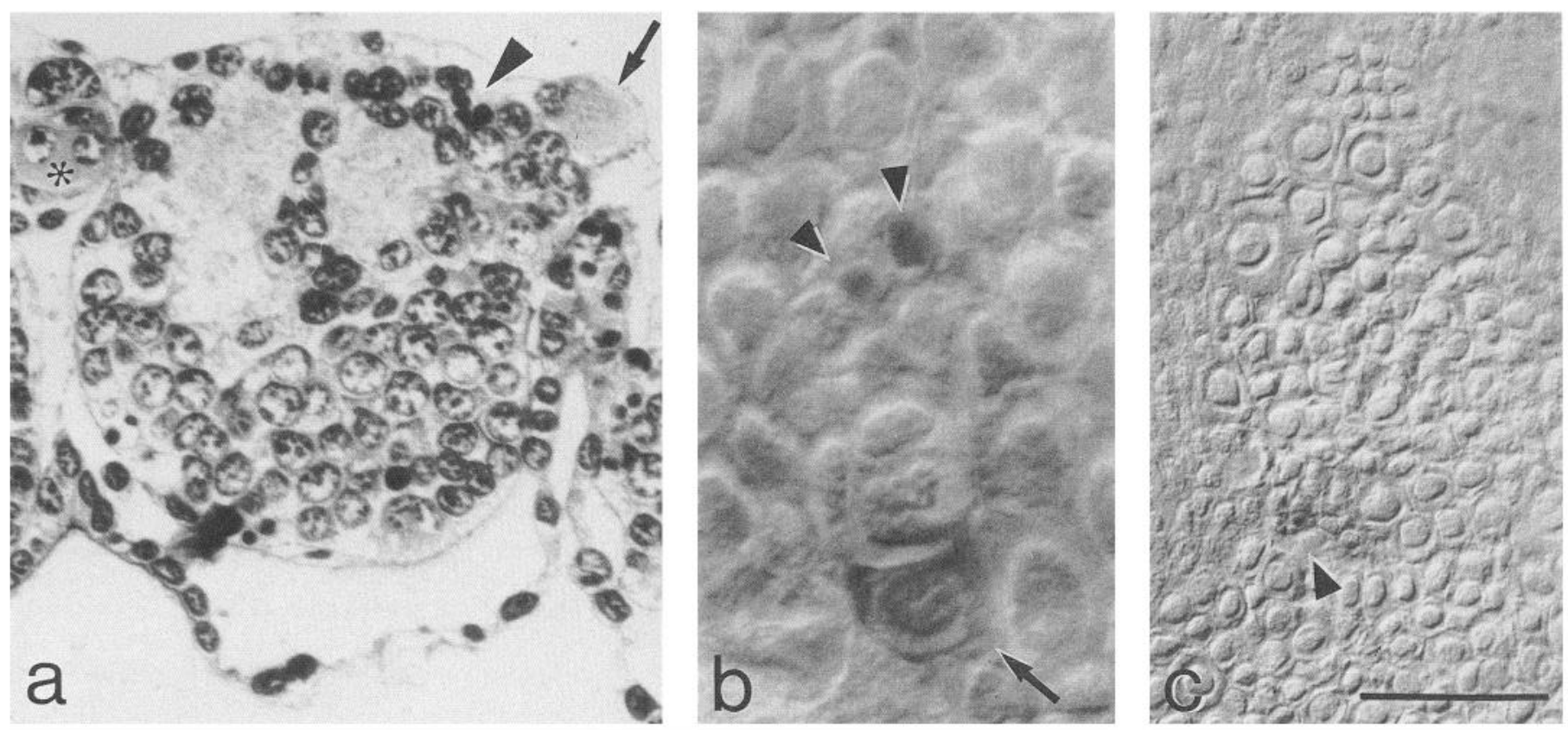

Figure 7. Histological preparations showing profiles of dying cells in MNB lineage groups. In $a$ a sagittal section of a $50 \%$ embryo shows the Al neuromere, with anterior to the left. The Al MNB (arrow) is dorsal, and is posterior to the profiles of three dying cells (arrowhead). Also seen is the T3 MNB (asterisk, upper left). Other pyknotic profiles are present in the section outside the MNB lineage group, including in the epidermis. In $b$ the T3 group of a 70\% embryo in whole-mount contains the MNB (arrow) and the strip of immediate progeny with the pyknotic dying cells (arrowheads) located distally, away from the MNB. In $c$ the degenerating MNB (arrowhead) is seen in a whole-mount of the T3 group in an embryo at just under $80 \%$ of development. The T3 MNB is situated posteriorly in its group of differentiating neural progeny. Scale bar: $50 \mu \mathrm{m}$ for $a$; 18 $\mu \mathrm{m}$ for $b ; 52 \mu \mathrm{m}$ for $c$.

death appeared at different times during development in the two segments, but in both the MNBs continued to display morphologies consistent with ongoing neural proliferation. The two temporally distinct episodes of cell death occurred when the rates of population increase deviated from linear in the two lineages (compare Fig. 6). These two periods also corresponded to the times during embryogenesis when the first and second embryonic cuticles underwent apolysis (asterisks in Fig. 10), in conjunction with embryonic molts. Apolysis of the entire first cuticle was apparent after $45 \%$ but by $50 \%$; apolysis of the second cuticle at $70 \% / 75 \%$ was most easily seen by examining the tips of limbs and cerci in whole-mounts. These times coincide with the appearance of molting in embryos (Bentley et al., 1979; Shankland and Bentley, 1983).

\section{Discussion}

In common with many other animals, adult insects have regional or segmental differences of neuronal number and type within the CNS. Differences are particularly obvious when thoracic neuromeres are compared with abdominal neuromeres, the thoracic neuromeres typically having at least a four- to fivefold greater number of neurons. In hemimetabolous insects such as the grasshopper Schistocerca, segmental differences are established during embryogenesis. Neuroblasts of the ventral nerve cord die before larval emergence, and during subsequent larval development, no new neurons are born, and few are lost through cell death. In holometabolous insects such as Manduca and Drosophila, segmental differences are minor at the end of embryogenesis, but are elaborated during larval life. Some neuroblasts reenlarge and divide in segment-specific patterns; as well, there is selective death of progeny segment by segment.
Differential segmental development is the combined outcome of differential neuronal proliferation and differential cell death. Thoracic neuroblasts typically are longer lived and generate progeny at a higher rate than abdominal neuroblasts. In Schistocerca, embryonic thoracic and abdominal neuromeres initially have similar arrays of neuroblasts (Bate, 1976). Whereas twothirds of the thoracic neuroblasts are still present midway through embryonic development, only one-third of the abdominal neuroblasts remain (Shepherd and Bate, 1990). In Manduca and Drosophila, upward of 23 neuroblasts persist postembryonically in thoracic hemineuromeres, but only three or four persist in a typical abdominal hemineuromere (Booker and Truman, 1987; Truman and Bate, 1988). Moreover, thoracic neuroblasts generally divide at higher rates than do abdominal neuroblasts (Booker and Truman, 1987; Truman and Bate, 1988; Shepherd and Bate, 1990). Cell death contributes further to the disparity in neuronal number between thorax and abdomen. In Schistocerca, embryonic cell death is greater overall in abdominal than in thoracic neuromeres (Goodman and Bate, 1981); in Manduca, cell death at the larval to pupal transition is likewise greater in abdominal lineages than in comparable thoracic lineages (Booker and Truman, 1987).

The mature T3 midline group is shown here to contain on average 93 neurons, and the $\mathrm{Al}$ midline group, on average 59 neurons. The numerical difference is due in part to a $6 \%$ difference in MNB life-span: the T3 MNB is present for $49 \%$; the A1 MNB, for $43 \%$ of embryonic development. The MNB groups grow at the rate of 10 neurons per $5 \%$ of development (equivalent to five MNB divisions) except during episodes of cell death. Assuming that the MNBs continue to divide at the same rate throughout their life-spans, we estimate that about 98 progeny 


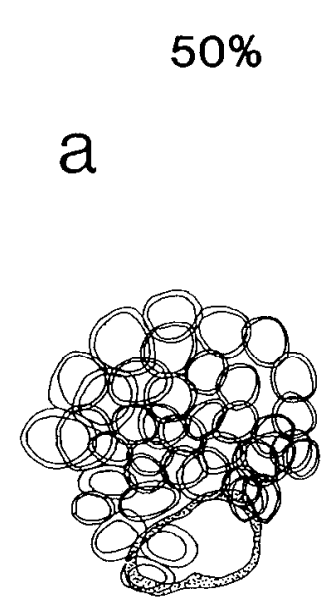

T3
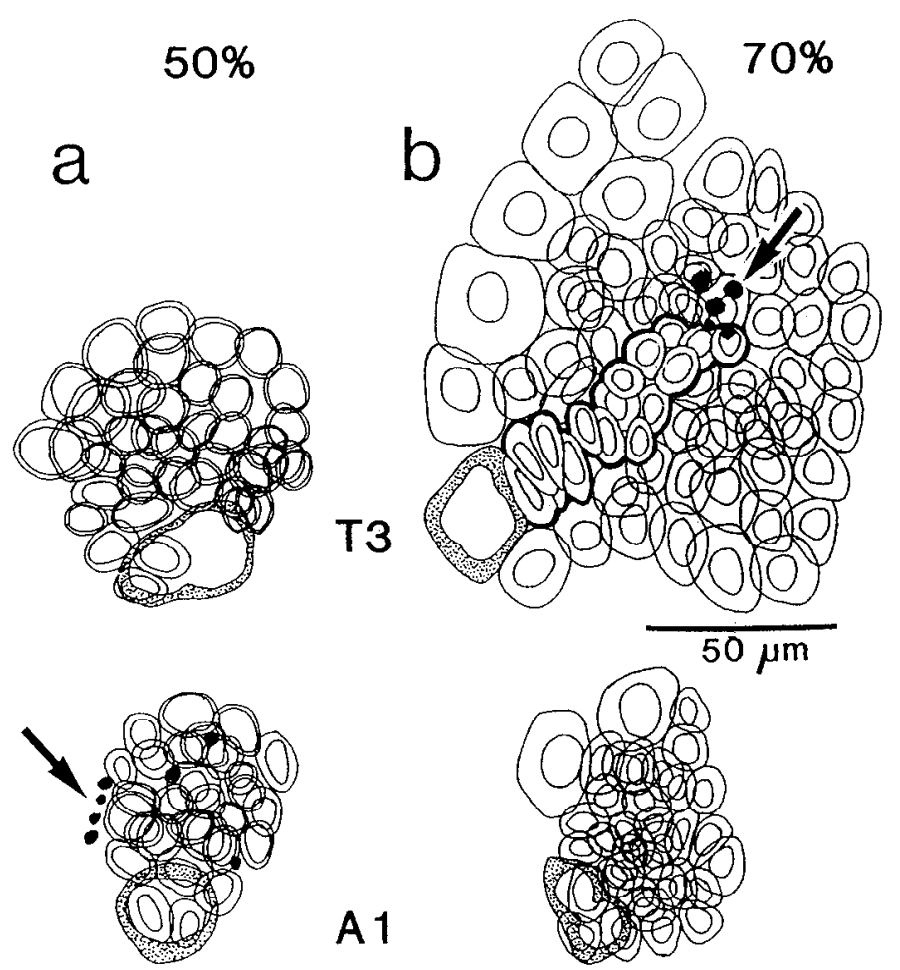

A1

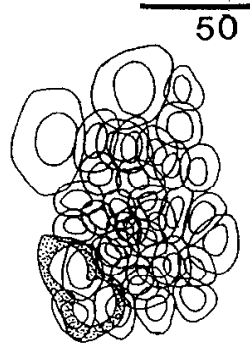

Figure 8. MNB lineage groups in segments $\mathrm{T} 3$ and $\mathrm{Al}$ at $50 \%(a)$ and $70 \%(b)$ of development showing timing of cell death. In $a$, from a $50 \%$ embryo, profiles of dying cells (some indicated by arrow) are present in the A1 group but not in the T3 group. In $b$, from a $70 \%$ embryo, dying cells (arrow) are observed in T3 but not A1. Nuclei of dying cells appeared as dense spots in toluidine blue-stained whole-mounts (camera lucida drawings). The MNB is located posteriorly in each group (stippled). The diagonal strip of the newest MNB progeny in the T3 group of $b$ is indicated by a bold outline. Dying cells occur at its anterior end (anterior is up).

are produced in the T3 lineage, whereas about 86 progeny (12 fewer) are produced in the A1 lineage. This agrees with earlier estimates (Goodman and Bate, 1981). Furthermore, more neurons die in $\mathrm{Al}$ than $\mathrm{T} 3$, further contributing to the numerical difference between the groups. Comparing the estimated number of progeny born with the number surviving there is a fivefold difference in cell death: 5 progeny die in T3 (98 born, 93 survive) whereas about 27 progeny die in A1 ( 86 born, 59 survive). Considering the relative numbers of pyknotic nuclei, the A1 peak at $50 \%$ is 3.6 times higher than the T3 peak at $70 \%$. The relative heights of the two peaks indicate that cell death is markedly greater in A1 than T3. Whether further differences in the number of progeny result from reduced rates of MNB division during plateau periods should be determined using a more direct measure of cell proliferation, for example, labeling with bromodeoxyuridine. Previous reports have stressed the role of either cell death (Goodman and Bate, 1981) or differential neuroblast division rates and life-span (Shepherd and Bate, 1990) in establishing segmental differences in neuron number. We suggest that cell death is predominant in shaping differences in cell number between the T3 and A1 MNB groups.

Our measure of division rates for the T3 MNB lineage compares closely with that of Shepherd and Bate (1990) for lineages of three other identified thoracic neuroblasts. They found that in each lineage 11-12 progeny were labeled during a $24 \mathrm{hr}$ pulse of bromodeoxyuridine (equivalent to $5 \%$ of development). The count overestimates the number of progeny produced during a given time, because ganglion cells are labeled prior to division. Shepherd and Bate (1990) accordingly estimated a rate of four to five neuroblast divisions per $24 \mathrm{hr}$, the upper number in agreement with our estimate. By contrast, their measurements show that unidentified abdominal neuroblasts in more posterior neuromeres have division rates about half that of thoracic ones (Shepherd and Bate, 1990). The Al neuromere is suggested to be transitional between the thoracic neuromeres and the more posterior abdominal neuromeres (Truman and Bate, 1988; Shepherd and Bate, 1990). Consistent with this idea are our findings that the A1 MNB shares the thoracic rate of cell division, but that the extent and timing of cell death among its progeny are clearly different from those of the T3 MNB.

Not only do different numbers of neurons die between segments, but different subpopulations of neurons in the MNB lineages are targeted. In T3, cell death occurs between $70 \%$ and $78 \%$ of embryogenesis, at a time when the last progeny are being produced in the lineage. The profiles of dying cells are spatially close to the last ganglion mother cells that arise from the MNB, suggesting that the dying cells are also among the last born of
Figure 9. Patterns of cell death in the T3 MNB lineage at $70 \%(a-c)$ and the Al MNB lineage at $50 \%(d, e)$ and $55 \%$ $(f)$ of development. The MNB (stippled), adjacent progeny, and the pyknotic nuclei in a group are drawn individually. The boundary of the entire group is outlined (anterior is up). In T3 at $70 \%$ the pyknotic profiles occur immediately adjacent to a strip of 10-12 progeny that are the most recent progeny of the MNB. The strip extends anteriorly in $a$, laterally in $b$, and curves ventrally in $c$. In $\mathrm{Al}$, the most recent progeny are closest to the MNB, but not as obviously delineated as T3. Pyknotic profiles occur throughout the group.
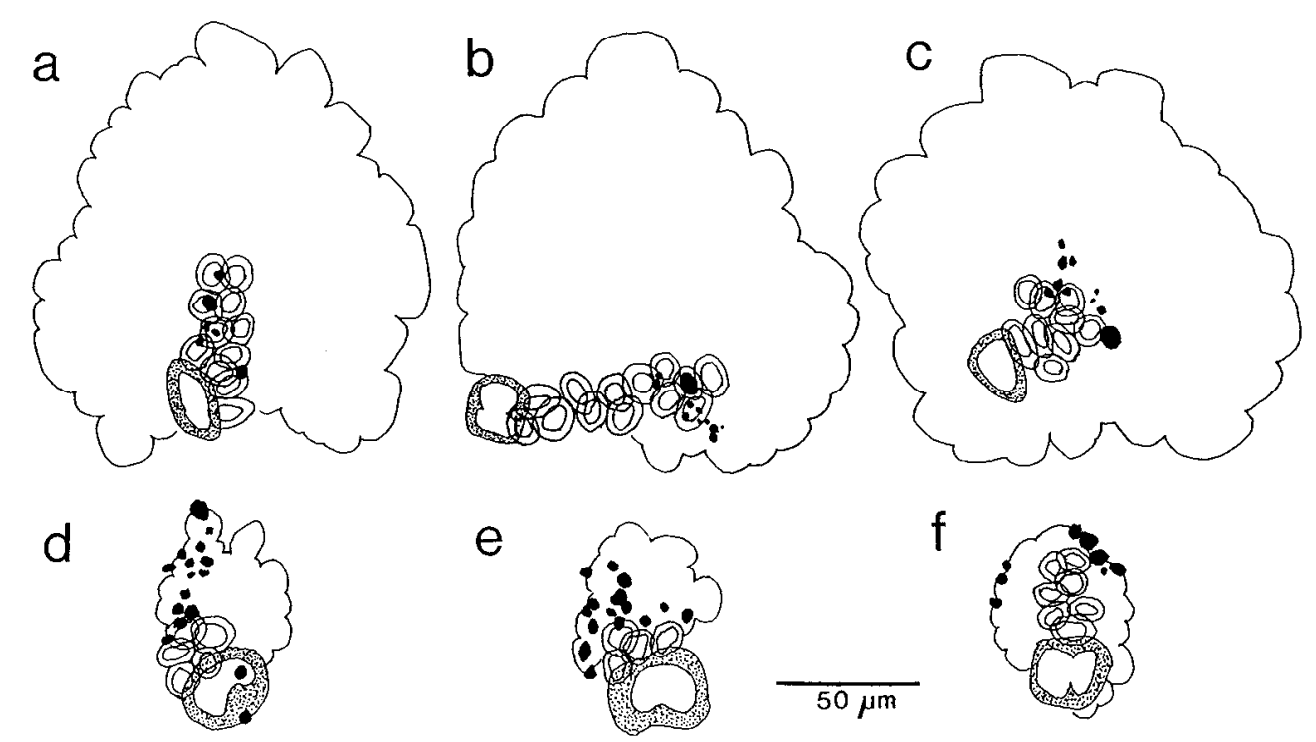

e

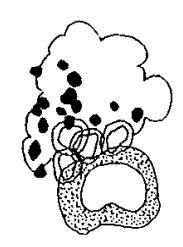

$50 \mu \mathrm{m}$

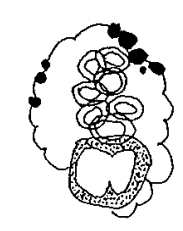


the lineage. By contrast, in Al cell death occurs between $45 \%$ and $60 \%$ of embryogenesis, beginning at a time when the MNB group contains perhaps 30 neurons, and the MNB is through only one-third of its life-span. In general, then, neurons produced earlier in the lineage are targeted for death in $\mathrm{Al}$, whereas those produced later are targeted for death in T3.

The mature T3 and $\Lambda 1$ midline groups differ in their complement of neuronal types. For example, the T3 group contains many more efferent neurons than does the Al group. Our data are consistent with the hypothesis that this is a further, specific, outcome of the selective cell death described above. This assumes that the MNB lineages are generally similar between segments, with the different types of neuron being produced in a stereotyped order. This would contrast with a situation, say, where MNB progeny assume their mature phenotypes irrespective of birth order, and where cell death only reduces the population of progeny to be specified. Available evidence is limited but favors the former model; in the MNB lineage and a few other lineages the earliest-born neurons, at least, arise in a reproducible sequence from particular ganglion mother cells (Goodman and Bate, 1981).

The earliest-born progeny in the MNB lineage are efferent neurons (Goodman and Bate, 1981). From a study of the adult T3 midline group, we have suggested, further, that three types of neuron are produced sequentially in the MNB lineage: efferents, local interneurons, and intersegmental interneurons (Thompson and Siegler, 1991). Given this sequence, the episode of cell death in A1 between $45 \%$ and $60 \%$ of embryogenesis would be expected to remove efferent and local interneurons. The midline group in A1 has 3 efferent neurons, as compared with about 20 in T3 (Siegler et al., 1991); cell death in the A1 group would be accounted for if 17 efferent neurons and perhaps 8 local interneurons are removed from the population. The targeting of local interneurons, which are almost exclusively auditory in function in $\mathrm{T} 3$, is plausible given the greatly reduced auditory projection in Al as compared with T3 (Halex et al., 1988; Thompson and Siegler, 1991). By contrast, the episode of cell death that is between $70 \%$ and $78 \%$ removes later-born neurons, suggested to be intersegmental interneurons. Cell death is limited in T3 compared with A1, and a large population of intersegmental interneurons remains. There are other examples in insects where apparently homologous neurons survive or die in a segment-specific manner (Bate et al., 1981; Loer et al., 1983; Truman, 1984; Weeks et al., 1992).

Cell death is increasingly being seen as an active, differentiative event in the development of the nervous system (Truman, 1987; Schwartz, 1991). Whereas some neurons survive and elaborate their unique characteristics of morphology and physiology, others begin a program of RNA and protein synthesis that ultimately leads to death. In systems amenable to genetic analysis, evidence is increasing that the alternative cell fates of survival or death are mutable. Mutations identified in Caenorhabditis elegans, for example, prevent the initiation of normal cell death or result in the inappropriate death of cells that otherwise survive (Ellis and Horvitz, 1986; Ellis et al., 1991; Driscoll and Chalfie, 1992).

Two questions are of interest relative to our results: what developmental mechanisms lead to segmental differences, and what developmental events trigger cell death? A possibility investigated previously to account for segmental differences is that the lack of appropriate peripheral muscle targets leads to the death of efferents in abdominal segments. Against this idea is

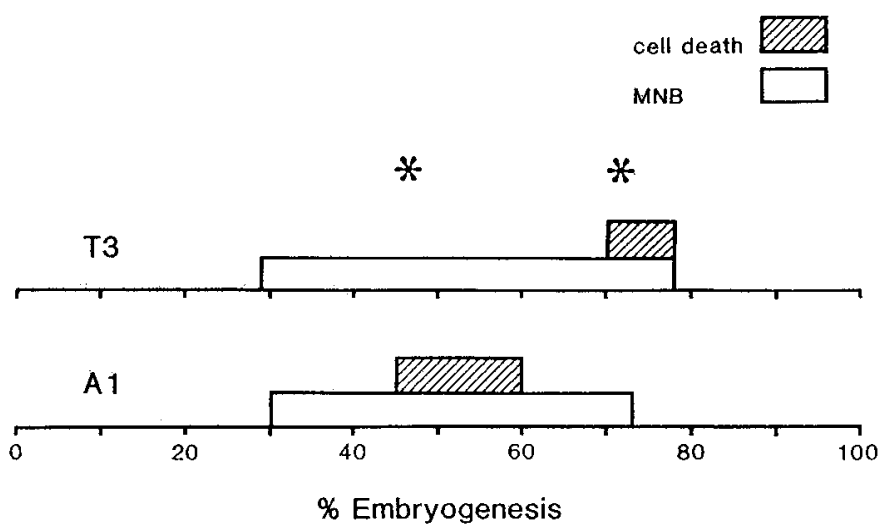

Figure 10. Phases of MNB proliferative activity and periods of cell death in segments T3 and Al during embryogenesis. The T3 MNB is born at $29 \%$ of development and dies at $78 \%$. The A1 MNB is born at $30 \%$ of development and dies at $73 \%$. An episode of cell death occurs between $70 \%$ and $78 \%$ of development in T3, and between $45 \%$ and $60 \%$ of development in A1. Asterisks at $45-50 \%$ and $70-75 \%$ indicate apolyses associated with molting of the first and second embryonic cuticles.

the finding that when the T3 limb bud is ablated early in embryogenesis, efferents normally innervating the limb nonetheless survive (Whitington et al., 1982). Moreover, as shown in the present study, cell death in the A1 midline group begins at $45 \%$, a time when the T3 MNB efferents are themselves still within the CNS and far from their peripheral targets (Myers et al., 1990). Thus, no differential peripheral cue is available at the appropriate time. A target-related mechanism would be plausible only if a key central target is postulated. Rather than environmental interactions being important, particular neurons might be spared or targeted for cell death as a result of intrinsic differences that occur segment by segment. Such differences might be conferred by segmentation or homeotic genes.

Several related observations lead us to suggest that the episodes of cell death during embryogenesis of Schistocerca are under hormonal control. Embryonic ecdysteroids, which are derived matcrnally, are storcd as inactive conjugates associated with yolk proteins; periodic metabolism of the conjugates is thought to underlie increases in the level of free ecdysteroids, and thus embryonic molts (reviewed by Hoffmann and Lagueux, 1985). An indicator of a change in ecdysteroid titer is the occurrence of apolysis, the separation of epidermis from overlying cuticle in preparation for the deposition of new cuticle. Studies of postembryonic molting in several insects indicate that apolysis occurs relatively late in the rising phase of increasing ecdysteroid titer, near the peak value (Riddiford, 1985). In Schistocerca, apolysis of the first embryonic cuticle occurs between $45 \%$ and $50 \%$; apolysis of the second, between $70 \%$ and $75 \%$ (Shankland and Bentley, 1983). The episodes of cell death we report are closely timed to these events and, we suggest, likewise triggered by rising ecdysteroid levels, much as ecdysteroids act in triggering metamorphic cell death in holometabolous insects (Truman, 1987).

Continued investigations of the elaboration of neural progeny from identified neuroblasts in the grasshopper can fill an important gap in our understanding of neural development. Specifically, studies could consider the mechanisms that control diversity of phenotype within a neural lineage, and the possible sources of flexibility in these events that allow for segmental and regional diversity within the CNS. By examining identified 
neuroblasts and their progeny, with the precision possible in the grasshopper, the cellular mechanisms can begin to be discovered.

\section{References}

Altman JS, Bell EM (1973) A rapid method for the demonstration of nerve cell bodies in invertebrate central nervous systems. Brain Res 63:487-489.

Bate CM (1976) Embryogenesis of an insect nervous system. I. A map of the thoracic and abdominal neuroblasts in Locusta migratoria. $\mathrm{J}$ Embryol Exp Morphol 35:107-123.

Bate CM, Grunewald EB (1981) Embryogenesis of an insect nervous system. II. A second class of neuronal precursor cells and the origin of the intersegmental connectives. J Embryol Exp Morphol 61:317330.

Bate M, Goodman CS, Spitzer NC (1981) Embryonic development of identified neurons: segment specific differences in the $\mathrm{H}$ cell homologs. J Neurosci 1:103-106.

Bentley D, Keshishian H, Shankland M, Toroian-Raymond A (1979) Quantitative staging of embryonic development of the grasshopper, Schistocerca nitens. J Embryol Exp Morphol 61:317-330.

Booker R, Truman JW (1987) Postembryonic neurogenesis in the CNS of the tobacco hornworm, Manduca sexta. I. Neuroblast arrays and the fate of their progeny during metamorphosis. J Comp Neurol 255:548-559.

Doe CQ, Goodman CS (1985) Early events in insect neurogenesis. II. The role of cell interactions and cell lineage in the determination of neuronal precursor cells. Dev Biol 111:206-219.

Doe CQ, Kuwada JY, Goodman CS (1985) From epithelium to neuroblasts to neurons: the role of cell interactions and cell lineage during insect neurogenesis. Philos Trans R Soc Lond [Biol] 312:67-81.

Driscoll M, Chalfie M (1992) Developmental and abnormal cell death in C. elegans. Trends Neurosci 15:15-19.

Ellis HM, Horvitz HR (1986) Genetic control of programmed cell death in the nematode C. elegans. Cell 44:817-829.

Ellis RE, Yuan J, Horvitz HR (1991) Mechanisms and functions of cell death. Annu Rev Cell Biol 7:663-698.

Evans PD, O'Shea M (1977) An octopaminergic neuron modulates neuromuscular transmission in the locust. Nature 270:257-259.

Evans PD, O'Shea M (1978) The identification of an octopaminergic neuron and the modulation of a myogenic rhythm in the locust. $J$ Exp Biol 73:235-260.

Evans PD, Siegler MVS (1982) Octopamine-mediated relaxation of maintained and catch tension in locust skeletal muscle. J Physiol (Lond) 324:93-112.

Goodman CS, Bate M (1981) Neuronal development in the grasshopper. Trends Neurosci 4:163-169.

Goodman CS, Spitzer NC (1979) Embryonic development of identified neurones: differentiation from neuroblast to neurone. Nature 280:208-214.

Halex H, Kaiser W, Kalmring K (1988) Projection areas and branching patterns of the tympanal receptor cells in migratory locusts, Locust migratoria and Schistocerca gregaria. Cell Tissue Res 253:517-528.

Hoffman JA, Lagueux M (1985) Endocrine aspects of embryonic development in insects. In: Comprehensive insect physiology, biochemistry and pharmacology, Vol 1 (Kerkut GA, Gilbert LI, eds), pp 435460. New York: Pergamon.

Hoyle G (1978) The dorsal unpaired neurons of the locust metathoracic ganglion. J Neurobiol 9:43-57.

Lange AB, Orchard I (1984) Dorsal unpaired median neurons and ventral bilaterally paired neurons project to a visceral muscle in an insect. J Neurobiol 15:441-453.

Loer CM, Steeves JD, Goodman CS (1983) Neuronal cell death in grasshopper embryos: variable patterns in different species, clutches, and clones. J Embryol Exp Morphol 78:169-182.

Malamud JG, Mizisin AP, Josephson RK (1988) The effects of oclopamine on contraction kinetics and power output of a locust light muscle. J Comp Physiol [A] 162:827-835.
McConnell SK (1991) The gencration of ncuronal diversity in the central nervous system. Annu Rev Neurosci 14:269-300.

Morton DB, Evans PD (1984) Octopamine release from an identified neurone in the locust. J Exp Biol 113:269-287.

Myers CM, Whitington PM, Ball EE (1990) Embryonic development of the innervation of the locust extensor tibiae muscle by identified neurons: formation and elimination of inappropriate axon branches. Dev Biol 137:194-206.

Riddiford LM (1985) Hormone action at the cellular level. In: Comprehensive insect physiology, biochemistry, and pharmacology, Vol 8 (Kerkut GA, Gilbert LI, eds), pp 37-84. New York: Pergamon.

Schwartz I.M (1991) The role of cell death genes during development. Bioessays 13:389-395.

Shankland M, Bentley D (1983) Sensory receptors, differentiation, and axonal pathfinding in the cercus of the grasshopper embryo. Dev Biol 97:468-482.

Shepherd D, Bate CM (1990) Spatial and temporal patterns of neurogenesis in the embryo of the locust (Schistocerca gregaria). Development 108:83-96.

Siegler MVS, Pousman CA (1990a) Distribution of motor neurons into anatomical groups in grasshopper metathoracic ganglion. J Comp Neurol 297:298-312.

Siegler MVS, Pousman CA (1990b) Motor neurons of grasshopper metathoracic ganglion occur in stereotypic anatomical groups. J Comp Neurol 297:313-327.

Siegler MVS, Manley PE Jr, Thompson KJ (1991) Sulphide silver staining for endogenous heavy metals reveals subsets of dorsal unpaired median (DUM) neurones in insects. J Exp Biol 157:565-571.

Stevenson PA, Pflüger H-J, Eckert M, Rapus J (1992) Octopamine immunoreactive cell populations in locust thoracic-abdominal nervous system. J Comp Neurol 315:382-397.

Taghert PH, Goodman CS (1984) Cell determination and differentiation of identified serotonin immunoreactive neurons in the grasshopper embryo. J Neurosci 4:980-1000.

Thompson KJ, Siegler MVS (1989) Properties of the small dorsal unpaired median (DUM) neurons of the grasshopper. Soc Neurosci Abstr 15:1296.

Thompson KJ, Siegler MVS (1991) Anatomy and physiology of spiking local and intersegmental interneurons in the median neuroblast lineage of the grasshopper. J Comp Neurol 305:659-675.

Truman JW (1984) Cell death in invertebrate nervous systems. Annu Rev Neurosci 7:171-188.

Truman JW (1987) The insect nervous system as a model system for the study of neuronal death. Curr Top Dev Biol 21:99-116.

Truman JW, Bate CM (1988) Spatial and temporal patterns of neurogenesis in the central nervous system of Drosophila melanogaster. Dev Biol 125:145-157.

Watson AHD (1984) The dorsal unpaired median neurons of the locust metathoracic ganglion: neuronal structure and diversity, and synapse distribution. J Neurocytol 13:303-327.

Weeks JC, Roberts WM, Trimble DL (1992) Hormonal regulation and segmental specificity of motoneuron phenotype during metamorphosis of the tobacco hornworm, Manduca sexta. Dev Biol 149: 185-196.

Wheeler WM (1893) A contribution to insect embryology. J Morphol $8: 1-160$.

Whim MD, Evans PD (1988) Octopaminergic modulation of flight muscle in the locust. J Exp Biol 134:247-266.

Whim MD, Evans PD (1989) Age-dependence of octopaminergic modulation of flight muscle in the locust. J Comp Physiol [A] 165: 125-137.

Whitington PM, Bate M, Seifert E, Ridge K, Goodman CS (1982) Survival and differentiation of identified embryonic neurons in the absence of their target muscles. Science 215:973-975.

Witten JL, Truman JW (1991) The regulation of transmitter expression in postembryonic lineages in the moth Manduca sexta. I. Transmitter identification and developmental acquisition of expression. J Neurosci 11:1980-1989. 